J. Environ. Sci.

Institute of Environmental Studies and Research - Ain Shams University

\title{
FREQUENCY OF SOME ENVIRONMENTAL FACTORS WITH POTENTIAL RELATIONS TO BREAST DISEASES AMONG A GROUP OF EGYPTIAN FEMALES
}

\author{
El Sobky, Abeer, F. ${ }^{(1)}$; El Bokhary, M. ${ }^{(1)}$; AwadAllah, Hala, I. $^{(1)}$ \\ and Abdel Hamid, Hanaa, A. ${ }^{(2)}$
}

1) Medical Sciences Department, Institute of Environmental Studies and Research, Ain Shams University 2) Radio-diagnosis Department, Faculty of Medicine, Ain Shams University

\begin{abstract}
This study was conducted to highlight the significant environmental factors that may have impacts on breast benign and malignant diseases. This study was carried out on one hundred females who came to radiology department to perform mammographic examination. A complete personal and family history of patients was taken, clinical examination was done, all cases were asked to fill a questionnaire about how often they deal with each item of the environmental factors including (alcohol, caffeine, smoking, use of some material that are commonly used in daily life and known to have estrogenic effect including (food and soda cans, plastic containers, insecticides, detergents and cleaning agent, deodorants and cosmetics) then digital mammographic examination was done for all cases. Cases with high $17 \mathrm{~b}$ Estradiol hormone blood level were excluded from the study. The quantitative data were presented as mean and standard deviations. Also qualitative variables were presented as number and percentages. The comparison between groups regarding qualitative data was done by using Chi-square test while the comparison between more than two groups with quantitative data were done by using One Way ANOVA. The confidence interval was set to $95 \%$ and the margin of error accepted was set to $5 \%$. So, the p-value was considered significant at the level of $<0.05$. Occurrence of benign and malignant lesions, breast calcifications and axillary lymphadenopathy was significantly associated to environmental estrogens containing items namely; use of diet \& soda cans, use of insecticides, use of detergents \& cleaning agents, use of deodorants, use of
\end{abstract}


cosmetics and use of plastic containers. Age, BMI of the patient and previous mammographic examinations was significantly associated to occurrence of benign and malignant lesions, breast calcifications and axillary lymphadenopathy. No significant association found between alcohol intake and axillary lymphadenopathy and between caffeine intake and breast calcifications. Caffeine intake, alcohol intake and smoking had no significant association with occurrence of benign and malignant lesions.

Keywords: Environmental estrogens, breast disease, benign breast lesions, digital mammography, breast calcification, breast cancer.

\section{INTRODUCTION}

Breast disease in women encompasses a spectrum of benign and malignant disorders (Morrow, 2000). Benign breast disease (BBD) has a high prevalence and a noticeable impact on women's quality of life (Friedenreich et al., 2000). In less-developed countries, breast cancer is the leading cause of cancer death in women; in developed countries, however, it has been surpassed by lung cancer as a cause of cancer death in women. In United States, breast cancer accounts for $29 \%$ of all cancers in women and is second only to lung cancer as a cause of cancer deaths. Several risk factors have been found to be clinically useful for assessing a patient's risk of breast cancer. Many of these factors form the basis of breast cancer risk assessment tools currently being used in the practice (Chalasani, 2017).

There are several breast imaging modalities available such as Ultrasound, CT, Digital Mammography, MRI and scintimammography. Mammograms are X-ray images of the breast. The images can be captured on film or stored directly onto a computer (digital). The aim of mammography is to obtain an optimum image along with maximum breast tissue visualization (Popli et al., 2014). 
A potential risk factor for breast cancer is exposure to environmental estrogens, a group of synthetic substances found in the environment that, when absorbed into a person's system, function in a similar way to estrogen. Estrogen stimulates breast cell growth, and exposure to estrogen over long periods of time, without any breaks, can increase the risk of breast cancer (Kane, 2013). Environmental estrogens are connected to everything from PMS (Premenstrual Syndrome) to cancer and reproductive problems in animals. In fact, environmental estrogens have been found to change our genes and give our bodies the instructions to produce cancer (Evans, 2009).

The present study was designed as a cross- sectional study in 2015 and 2016 to detect the association of exposure to some environmental factors (with more concern to those related to environmental estrogens) with different breast pathologies especially malignant breast diseases, aiming to reduce risk of breast cancer as much as possible.

\section{SUBJECTS AND METHODS}

The present study was designed as a cross- sectional study. It was conducted from March 2015 to July 2016 on one hundred females who came to radiology department to perform mammographic examination; this was done in Central Hospital Egypt (Nasr City next to City stars).

A standardized epidemiological questionnaire including age, smoking status, alcohol use, and family history of cancer was used to collect personal data through in-person interviews. 


\section{Inclusion criteria are:}

- Patient of known breast disease who came for follow up.

- Patients who came for regular check up.

- Patient who discovered breast abnormality during self-examination or medical assessment.

\section{Exclusion criteria are:}

- Taking any hormonal treatment.

- Having any endocrinal disease.

- Having high blood estrogen level.

- Lactating women.

- Lesions due to trauma.

- Females less than 40 years old.

A complete personal and family history of patients was taken. Clinical examination was done including inspection and palpation. All cases were asked to fill a questionnaire about how often they deal with each item as follows: Never (meaning that the patient never uses this item), occasionally (meaning that the patient uses this item once or twice per week) or daily (meaning that the patient uses this item every day). Items included in the questionnaire were alcohol, caffeine, smoking, use of some material that are commonly used in daily life and known to have estrogenic effect including (food and soda cans, plastic containers, insecticides, detergents and cleaning agent, deodorants and cosmetics).

Digital mammographic examination: Mammography was done for all cases as follows: One breast at a time was rested on a flat surface that contains the $\mathrm{X}$-ray plate. A compressor was pressed firmly against the breast to help 
flatten out the breast tissue. The X-ray picture was taken while patient is holding her breath. Routine views were obtained: (Cranio-caudal view -top to bottom-and Medio- lateral oblique) view.

Laboratory examination: Blood samples were collected for testing $17 \mathrm{~b}$ Estradiol hormone at the follicular phase of the menstrual cycle. Cases with high 17b Estradiol hormone blood level were excluded from the study.

Statistical analysis: Data were collected, revised, coded and entered to the Statistical Package of Social Science (IBM SPSS) version 23. The quantitative data were presented as mean and standard deviations. Also qualitative variables were presented as number and percentages. The comparison between groups regarding qualitative data was done by using Chi-square test while the comparison between more than two groups with quantitative data were done by using One Way ANOVA. The confidence interval was set to $95 \%$ and the margin of error accepted was set to $5 \%$. So, the p-value was considered significant at the level of $<0.05$.

\section{RESULTS}

\section{Breast abnormalities seen in digital mammography were as follows:}

a) Breast calcifications: The percentage of cases that showed no calcifications in mammography was $34 \%$, typically benign calcifications was $49 \%$ and cases showed suspicious calcifications was $17 \%$ as shown in figure (1).

b) Enlarged axillary lymph nodes: Cases that showed no lymph nodal enlargement in mammography was $36 \%$, inflammatory lymph nodal 
enlargement was $56 \%$ and malignant lymph nodal enlargement was $8 \%$ as shown in figure (2).

c) Benign and malignant breast lesions: Cases that categorized as BIRADS II (benign lesions) in mammography was $51 \%$, cases categorized as BIRADS III (probably benign lesions) in mammography was $14 \%$, cases categorized as BIRADS IV (probably malignant lesions) was $17 \%$ and cases categorized as BIRADS V (high suspicion of malignancy) was $18 \%$ as shown in figure (3).

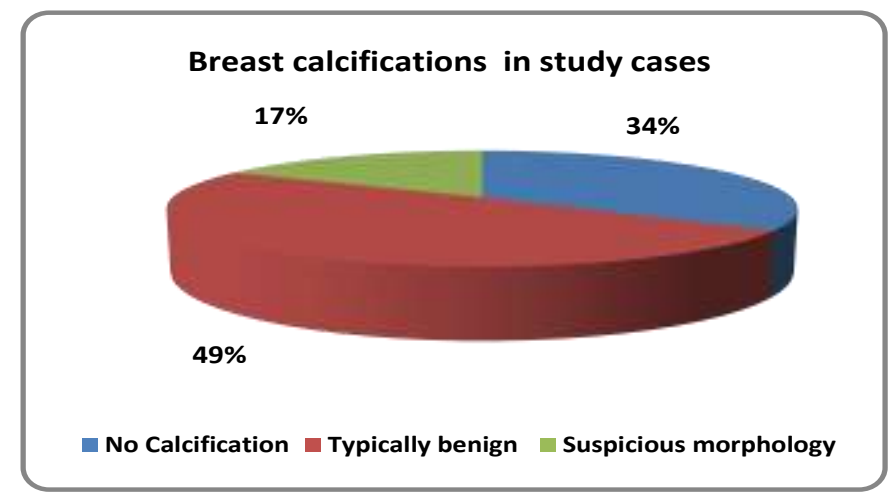

Figure (1): Percentage of breast calcifications in study cases

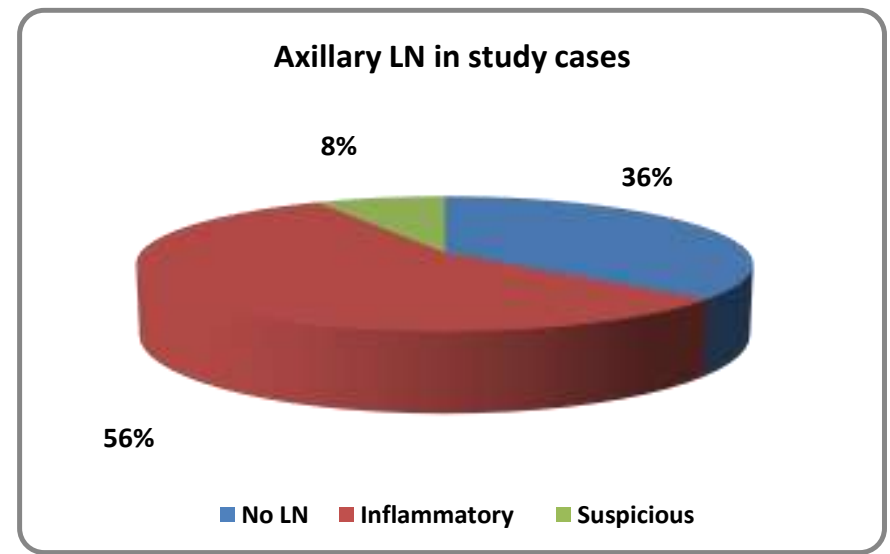

Figure (2): Percentage of axillary lymphadenopathy in study cases 


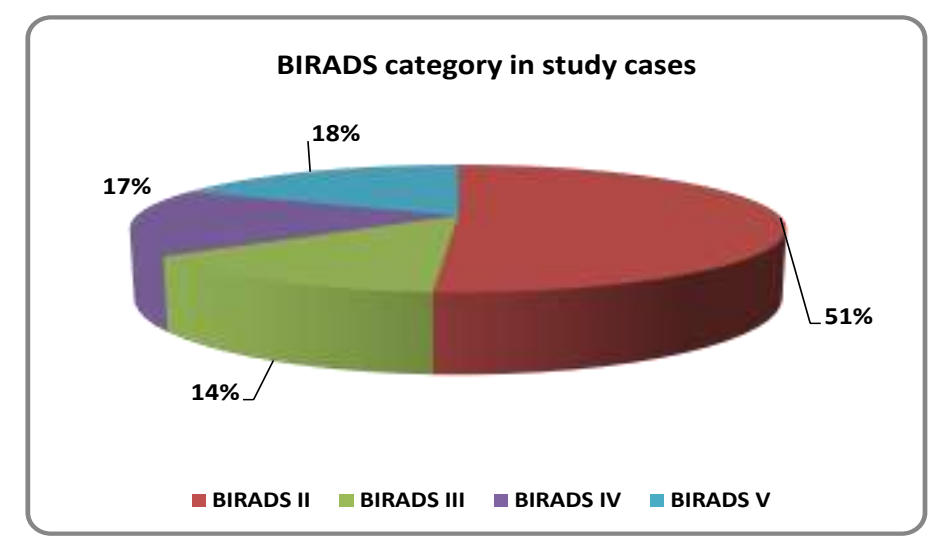

Figure (3): Percentage of different BIRADS categories in study cases

The association between the studied environmental factors and different pathological findings: Data were collected, revised, coded and entered to the Statistical Package for Social Science (IBM SPSS) version 23. The quantitative data were presented as mean and standard deviations. Also qualitative variables were presented as number and percentages. The comparison between groups regarding qualitative data was done by using Chi-square test while the comparison between more than two groups with quantitative data were done by using One Way ANOVA. The confidence interval was set to $95 \%$ and the margin of error accepted was set to $5 \%$. So, the $\mathrm{p}$-value was considered significant at the level of $<0.05$.

Association between axillary lymphadenopathy and studied environmental factors: Datain table (1) showed that there was high significant association between axillary lymphadenopathy and age, BMI of the patient, smoking, previous mammographic examinations and occupational radiological exposure. All studied factors of exposure to xenoestrogens including use of soda cans, use of insecticides, use of cosmetics, use of 
deodorants, use of detergents and cleaning agents and use of plastic containers showed high significant association with axillary lymphadenopathy. Caffeine intake showed significant association with axillary lymphadenopathy. Meanwhile, no significant association was found between axillary lymphadenopathy and alcohol consumption.

\section{Association between breast calcification and studied environmental}

factors: Data in table (2) showed that there was high significant association between breast calcifications and age, BMI of the patient, smoking, previous mammographic examinations and occupational radiological exposure. All studied factors of exposure to xenoestrogens including use of soda cans, use of insecticides, use of cosmetics, use of deodorants, use of detergents and cleaning agents and use of plastic containers showed high significant association with breast calcifications. Alcohol consumption showed significant association with breast calcifications. Meanwhile, no significant association was found between breast calcifications and caffeine intake.

\section{Association between benign and malignant lesions (according to BIRADS} classification) and studied environmental factors: Data in table (3) showed that there was high significant association between occurrence of benign and malignant lesions and age, BMI of the patient and previous mammographic examinations. All studied factors of exposure to xenoestrogens including use of soda cans, use of insecticides, use of cosmetics, use of deodorants and use of detergents and cleaning agents showed high significant association with axillary lymphadenopathy. Occurrence of benign and malignant lesions had significant association with occupational radiological exposure and use of plastic containers. Meanwhile, no significant association was found between 
J. Environ. Sci.

Institute of Environmental Studies and Research - Ain Shams University

occurrence of benign and malignant lesions and alcohol consumption, Caffeine intake and smoking.

Table (1): Association between studied environmental factors and axillary lymphadenopathy

\begin{tabular}{|c|c|c|c|c|c|c|c|}
\hline & \multicolumn{3}{|c|}{ Axill LN } & \multirow{3}{*}{ Test value } & \multirow{3}{*}{ P-value } & \multirow{3}{*}{ Sig. } \\
\hline & & \multirow{2}{*}{$\begin{array}{c}\text { Negative } \\
\text { No. }=18\end{array}$} & \multirow{2}{*}{$\begin{array}{c}\text { Benign } \\
\text { No. }=28\end{array}$} & \multirow{2}{*}{$\begin{array}{c}\text { Malignant } \\
\text { No. }=4\end{array}$} & & & \\
\hline & & & & & & & \\
\hline Age & $\begin{array}{c}\text { Mean } \pm \text { SD } \\
\text { Range }\end{array}$ & $\begin{array}{c}52.00 \pm 4.06 \\
40-56\end{array}$ & $\begin{array}{c}52.93 \pm 6.45 \\
40-61\end{array}$ & $\begin{array}{c}65.00 \pm 1.83 \\
63-67\end{array}$ & 9.628 & 0 & HS \\
\hline BMI & $\begin{array}{c}\text { Mean } \pm \text { SD } \\
\text { Range } \\
\end{array}$ & $\begin{array}{c}31.79 \pm 3.99 \\
24.34-35.61 \\
\end{array}$ & $\begin{array}{r}33.76 \pm 2.47 \\
27.69-36.57 \\
\end{array}$ & $\begin{array}{l}39.99 \pm 2.31 \\
37.2-42.02 \\
\end{array}$ & 11.611 & 0 & HS \\
\hline Alcohol & $\begin{array}{c}\text { No exposure } \\
\text { Mild expousre } \\
\text { Severe exposure }\end{array}$ & $\begin{array}{c}15(83.3 \%) \\
3(16.7 \%) \\
0(0.0 \%) \\
\end{array}$ & $\begin{array}{c}28(100.0 \%) \\
0(0.0 \%) \\
0(0.0 \%)\end{array}$ & $\begin{array}{c}4(100.0 \%) \\
0(0.0 \%) \\
0(0.0 \%) \\
\end{array}$ & 5.674 & 0.059 & NS \\
\hline Caffeine & $\begin{array}{c}\text { No exposure } \\
\text { Mild expousre } \\
\text { Severe exposure }\end{array}$ & $\begin{array}{l}5(27.8 \%) \\
5(27.8 \%) \\
8(44.4 \%) \\
\end{array}$ & $\begin{array}{l}14(50.0 \%) \\
9(32.1 \%) \\
5(17.9 \%) \\
\end{array}$ & $\begin{array}{c}2(50.0 \%) \\
0(0.0 \%) \\
2(50.0 \%) \\
\end{array}$ & 5.808 & 0.214 & $\mathbf{s}$ \\
\hline Smoking & $\begin{array}{c}\text { No exposure } \\
\text { Mild expousre } \\
\text { Severe exposure } \\
\end{array}$ & $\begin{array}{c}18(100.0 \%) \\
0(0.0 \%) \\
0(0.0 \%) \\
\end{array}$ & $\begin{array}{c}15(53.6 \%) \\
9(32.1 \%) \\
4(14.3 \%) \\
\end{array}$ & $\begin{array}{c}0(0.0 \%) \\
0(0.0 \%) \\
4(100.0 \%) \\
\end{array}$ & 34.091 & $\mathbf{0}$ & HS \\
\hline Previous mammogr & $\begin{array}{c}\text { No exposure } \\
\text { Mild expousre } \\
\text { Severe exposure } \\
\end{array}$ & $\begin{array}{c}14(77.8 \%) \\
4(22.2 \%) \\
0(0.0 \%) \\
\end{array}$ & $\begin{array}{c}17(60.7 \%) \\
11(39.3 \%) \\
0(0.0 \%) \\
\end{array}$ & $\begin{array}{c}1(25.0 \%) \\
0(0.0 \%) \\
3(75.0 \%) \\
\end{array}$ & 38.399 & $\mathbf{0}$ & HS \\
\hline Occupation rad expo & $\begin{array}{c}\text { No exposure } \\
\text { Mild expousre } \\
\text { Severe exposure } \\
\end{array}$ & $\begin{array}{c}18(100.0 \%) \\
0(0.0 \%) \\
0(0.0 \%)\end{array}$ & $\begin{array}{c}28(100.0 \%) \\
0(0.0 \%) \\
0(0.0 \%)\end{array}$ & $\begin{array}{c}0(0.0 \%) \\
3(75.0 \%) \\
1(25.0 \%) \\
\end{array}$ & 50 & $\mathbf{0}$ & HS \\
\hline Soda cans & $\begin{array}{c}\text { No exposure } \\
\text { Mild expousre } \\
\text { Severe exposure }\end{array}$ & $\begin{array}{c}0(0.0 \%) \\
0(0.0 \%) \\
18(100.0 \%)\end{array}$ & $\begin{array}{c}0(0.0 \%) \\
24(85.7 \%) \\
4(14.3 \%)\end{array}$ & $\begin{array}{l}2(50.0 \%) \\
2(50.0 \%) \\
0(0.0 \%)\end{array}$ & 58.691 & $\mathbf{0}$ & HS \\
\hline Plastic containers & $\begin{array}{c}\text { No exposure } \\
\text { Mild expousre } \\
\text { Severe exposure } \\
\end{array}$ & $\begin{array}{c}0(0.0 \%) \\
0(0.0 \%) \\
18(100.0 \%) \\
\end{array}$ & $\begin{array}{c}0(0.0 \%) \\
0(0.0 \%) \\
28(100.0 \%) \\
\end{array}$ & $\begin{array}{c}0(0.0 \%) \\
3(75.0 \%) \\
1(25.0 \%) \\
\end{array}$ & 36.702 & 0 & HS \\
\hline Detr. Clean & $\begin{array}{c}\text { No exposure } \\
\text { Mild expousre } \\
\text { Severe exposure }\end{array}$ & $\begin{array}{c}0(0.0 \%) \\
0(0.0 \%) \\
18(100.0 \%)\end{array}$ & $\begin{array}{c}0(0.0 \%) \\
14(50.0 \%) \\
14(50.0 \%)\end{array}$ & $\begin{array}{c}2(50.0 \%) \\
2(50.0 \%) \\
0(0.0 \%)\end{array}$ & 39.063 & 0 & HS \\
\hline Insecticides & $\begin{array}{c}\text { No exposure } \\
\text { Mild expousre } \\
\text { Severe exposure }\end{array}$ & $\begin{array}{c}0(0.0 \%) \\
0(0.0 \%) \\
18(100.0 \%)\end{array}$ & $\begin{array}{c}3(10.7 \%) \\
23(82.1 \%) \\
2(7.1 \%)\end{array}$ & $\begin{array}{c}2(50.0 \%) \\
2(50.0 \%) \\
0(0.0 \%)\end{array}$ & 48.357 & $\mathbf{0}$ & HS \\
\hline Cosmetics & $\begin{array}{c}\text { No exposure } \\
\text { Mild expousre } \\
\text { Severe exposure }\end{array}$ & $\begin{array}{c}12(66.7 \%) \\
6(33.3 \%) \\
0(0.0 \%)\end{array}$ & $\begin{array}{c}2(7.1 \%) \\
23(82.1 \%) \\
3(10.7 \%)\end{array}$ & $\begin{array}{c}0(0.0 \%) \\
0(0.0 \%) \\
4(100.0 \%)\end{array}$ & 45.971 & 0 & HS \\
\hline Deodrants & $\begin{array}{c}\text { No exposure } \\
\text { Mild expousre } \\
\text { Severe exposure }\end{array}$ & $\begin{array}{c}18(100.0 \%) \\
0(0.0 \%) \\
0(0.0 \%)\end{array}$ & $\begin{array}{c}2(7.1 \%) \\
18(64.3 \%) \\
8(28.6 \%)\end{array}$ & $\begin{array}{c}0(0.0 \%) \\
0(0.0 \%) \\
4(100.0 \%)\end{array}$ & 53.69 & 0 & HS \\
\hline
\end{tabular}


El Sobky, Abeer et al.

Table (2): Association between studied environmental factors and breast calcifications

\begin{tabular}{|c|c|c|c|c|c|c|c|}
\hline & \multicolumn{3}{|c|}{ Calcifications } & \multirow{3}{*}{ Test value } & \multirow{3}{*}{ P-value } & \multirow{3}{*}{ Sig. } \\
\hline & & Negative & Benign & Malignant & & & \\
\hline & & No. $=17$ & No. $=24$ & No. $=9$ & & & \\
\hline Age & $\begin{array}{c}\text { Mean } \pm S D \\
\text { Ranqe }\end{array}$ & $\begin{array}{c}51.76 \pm 4.05 \\
40-56\end{array}$ & $\begin{array}{c}52.29 \pm 6.45 \\
40-60\end{array}$ & $\begin{array}{c}60.33 \pm 5.74 \\
49-67\end{array}$ & 8.018 & 0.001 & HS \\
\hline BMI & $\begin{array}{c}\text { Mean } \pm S D \\
\text { Range }\end{array}$ & $\begin{array}{c}31.89 \pm 4.09 \\
24.34-35.61\end{array}$ & $\begin{array}{c}33.64 \pm 2.65 \\
27.69-36.33\end{array}$ & $\begin{array}{c}36.44 \pm 3.92 \\
32.13-42.02\end{array}$ & 5.205 & 0.009 & HS \\
\hline Alcohol & $\begin{array}{c}\text { No exposure } \\
\text { Mild expousre } \\
\text { Severe exposure }\end{array}$ & $\begin{array}{c}14(82.4 \%) \\
3(17.6 \%) \\
0(0.0 \%)\end{array}$ & $\begin{array}{c}24(100.0 \%) \\
0(0.0 \%) \\
0(0.0 \%)\end{array}$ & $\begin{array}{c}9(100.0 \%) \\
0(0.0 \%) \\
0(0.0 \%)\end{array}$ & 6.195 & 0.045 & S \\
\hline Caffeine & $\begin{array}{l}\text { No exposure } \\
\text { Mild expousre } \\
\text { Severe exposure }\end{array}$ & $\begin{array}{l}5(29.4 \%) \\
5(29.4 \%) \\
7(41.2 \%)\end{array}$ & $\begin{array}{l}14(58.3 \%) \\
7(29.2 \%) \\
3(12.5 \%)\end{array}$ & $\begin{array}{l}2(22.2 \%) \\
2(22.2 \%) \\
5(55.6 \%)\end{array}$ & 8.252 & 0.083 & NS \\
\hline Smoking & $\begin{array}{c}\text { No exposure } \\
\text { Mild expousre } \\
\text { Severe exposure }\end{array}$ & $\begin{array}{c}17(100.0 \%) \\
0(0.0 \%) \\
0(0.0 \%)\end{array}$ & $\begin{array}{l}14(58.3 \%) \\
7(29.2 \%) \\
3(12.5 \%)\end{array}$ & $\begin{array}{l}2(22.2 \%) \\
2(22.2 \%) \\
5(55.6 \%) \\
\end{array}$ & 22.321 & 0 & HS \\
\hline $\begin{array}{l}\text { Previous } \\
\text { mammogr }\end{array}$ & $\begin{array}{c}\text { No exposure } \\
\text { Mild expousre } \\
\text { Severe exposure }\end{array}$ & $\begin{array}{c}14(82.4 \%) \\
3(17.6 \%) \\
0(0.0 \%)\end{array}$ & $\begin{array}{c}12(50.0 \%) \\
12(50.0 \%) \\
0(0.0 \%)\end{array}$ & $\begin{array}{l}6(66.7 \%) \\
0(0.0 \%) \\
3(33.3 \%) \\
\end{array}$ & 22.071 & 0 & HS \\
\hline $\begin{array}{c}\text { Occupation } \\
\text { rad expo }\end{array}$ & $\begin{array}{c}\text { No exposure } \\
\text { Mild expousre } \\
\text { Severe exposure }\end{array}$ & $\begin{array}{c}17(100.0 \%) \\
0(0.0 \%) \\
0(0.0 \%)\end{array}$ & $\begin{array}{c}24(100.0 \%) \\
0(0.0 \%) \\
0(0.0 \%)\end{array}$ & $\begin{array}{l}5(55.6 \%) \\
3(33.3 \%) \\
1(11.1 \%)\end{array}$ & 19.807 & 0.001 & HS \\
\hline Soda cans & $\begin{array}{c}\text { No exposure } \\
\text { Mild expousre } \\
\text { Severe exposure }\end{array}$ & $\begin{array}{c}0(0.0 \%) \\
0(0.0 \%) \\
17(100.0 \%)\end{array}$ & $\begin{array}{c}0(0.0 \%) \\
19(79.2 \%) \\
5(20.8 \%)\end{array}$ & $\begin{array}{c}2(22.2 \%) \\
7(77.8 \%) \\
0(0.0 \%)\end{array}$ & 41.511 & 0 & HS \\
\hline $\begin{array}{l}\text { Plastic } \\
\text { containers }\end{array}$ & $\begin{array}{c}\text { No exposure } \\
\text { Mild expousre } \\
\text { Severe exposure }\end{array}$ & $\begin{array}{c}0(0.0 \%) \\
0(0.0 \%) \\
17(100.0 \%)\end{array}$ & $\begin{array}{c}0(0.0 \%) \\
0(0.0 \%) \\
24(100.0 \%)\end{array}$ & $\begin{array}{l}0(0.0 \%) \\
3(33.3 \%) \\
6(66.7 \%) \\
\end{array}$ & 14.539 & 0.001 & HS \\
\hline Detr. Clean & $\begin{array}{c}\text { No exposure } \\
\text { Mild expousre } \\
\text { Severe exposure }\end{array}$ & $\begin{array}{c}0(0.0 \%) \\
0(0.0 \%) \\
17(100.0 \%)\end{array}$ & $\begin{array}{c}0(0.0 \%) \\
14(58.3 \%) \\
10(41.7 \%)\end{array}$ & $\begin{array}{l}2(22.2 \%) \\
2(22.2 \%) \\
5(55.6 \%)\end{array}$ & 25.434 & 0 & HS \\
\hline Insecticides & $\begin{array}{c}\text { No exposure } \\
\text { Mild expousre } \\
\text { Severe exposure }\end{array}$ & $\begin{array}{c}0(0.0 \%) \\
0(0.0 \%) \\
17(100.0 \%)\end{array}$ & $\begin{array}{c}0(0.0 \%) \\
21(87.5 \%) \\
3(12.5 \%)\end{array}$ & $\begin{array}{l}5(55.6 \%) \\
4(44.4 \%) \\
0(0.0 \%) \\
\end{array}$ & 61.521 & 0 & HS \\
\hline Cosmetics & $\begin{array}{c}\text { No exposure } \\
\text { Mild expousre } \\
\text { Severe exposure }\end{array}$ & $\begin{array}{c}12(70.6 \%) \\
5(29.4 \%) \\
0(0.0 \%)\end{array}$ & $\begin{array}{c}2(8.3 \%) \\
22(91.7 \%) \\
0(0.0 \%)\end{array}$ & $\begin{array}{l}0(0.0 \%) \\
2(22.2 \%) \\
7(77.8 \%) \\
\end{array}$ & 57.808 & 0 & HS \\
\hline Deodrants & $\begin{array}{c}\text { No exposure } \\
\text { Mild expousre } \\
\text { Severe exposure }\end{array}$ & $\begin{array}{c}17(100.0 \%) \\
0(0.0 \%) \\
0(0.0 \%)\end{array}$ & $\begin{array}{c}3(12.5 \%) \\
18(75.0 \%) \\
3(12.5 \%)\end{array}$ & $\begin{array}{c}0(0.0 \%) \\
0(0.0 \%) \\
9(100.0 \%)\end{array}$ & 70 & 0 & HS \\
\hline
\end{tabular}


J. Environ. Sci.

Institute of Environmental Studies and Research - Ain Shams University

Table (3): Association between studied environmental factors and occurrence of benign and malignant breast lesions

\begin{tabular}{|c|c|c|c|c|c|c|}
\hline & & \multicolumn{2}{|c|}{ BIRADS } & \multirow{3}{*}{ Test value } & \multirow{3}{*}{ P-value } & \multirow{3}{*}{ Sig } \\
\hline & & \multirow{2}{*}{$\begin{array}{c}\text { Benign } \\
\text { No. }=32 \\
\end{array}$} & \multirow{2}{*}{$\begin{array}{c}\text { Malignant } \\
\text { No. }=18\end{array}$} & & & \\
\hline & & & & & & \\
\hline Age & $\begin{array}{c}\text { Mean } \pm S D \\
\text { Range }\end{array}$ & $\begin{array}{c}51.72 \pm 5.16 \\
40-57\end{array}$ & $\begin{array}{c}56.83 \pm 7.13 \\
43-67\end{array}$ & -2.926 & 0.005 & HS \\
\hline BMI & $\begin{array}{c}\text { Mean } \pm S D \\
\text { Range }\end{array}$ & $\begin{array}{c}32.67 \pm 3.53 \\
24.34-36.33\end{array}$ & $\begin{array}{c}35.10 \pm 3.60 \\
29.72-42.02\end{array}$ & -2.317 & 0.025 & HS \\
\hline Alcohol & $\begin{array}{c}\text { No exposure } \\
\text { Mild expousre } \\
\text { Severe exposure }\end{array}$ & $\begin{array}{c}29(90.6 \%) \\
3(9.4 \%) \\
0(0.0 \%)\end{array}$ & $\begin{array}{c}18(100.0 \%) \\
0(0.0 \%) \\
0(0.0 \%)\end{array}$ & 1.795 & 0.18 & NS \\
\hline Caffeine & $\begin{array}{c}\text { No exposure } \\
\text { Mild expousre } \\
\text { Severe exposure }\end{array}$ & $\begin{array}{l}11(34.4 \%) \\
11(34.4 \%) \\
10(31.3 \%)\end{array}$ & $\begin{array}{l}10(55.6 \%) \\
3(16.7 \%) \\
5(27.8 \%)\end{array}$ & 2.567 & 0.277 & NS \\
\hline Smoking & $\begin{array}{c}\text { No exposure } \\
\text { Mild expousre } \\
\text { Severe exposure }\end{array}$ & $\begin{array}{c}22(68.8 \%) \\
7(21.9 \%) \\
3(9.4 \%)\end{array}$ & $\begin{array}{l}11(61.1 \%) \\
2(11.1 \%) \\
5(27.8 \%)\end{array}$ & 3.282 & 0.194 & NS \\
\hline $\begin{array}{l}\text { Previous } \\
\text { mammogr }\end{array}$ & $\begin{array}{l}\text { No exposure } \\
\text { Mild expousre } \\
\text { Severe exposure }\end{array}$ & $\begin{array}{c}17(53.1 \%) \\
15(46.9 \%) \\
0(0.0 \%)\end{array}$ & $\begin{array}{c}15(83.3 \%) \\
0(0.0 \%) \\
3(16.7 \%)\end{array}$ & 15.413 & 0 & HS \\
\hline $\begin{array}{c}\text { Occupation rad } \\
\text { expo }\end{array}$ & $\begin{array}{l}\text { No exposure } \\
\text { Mild expousre } \\
\text { Severe exposure }\end{array}$ & $\begin{array}{c}32(100.0 \%) \\
0(0.0 \%) \\
0(0.0 \%)\end{array}$ & $\begin{array}{c}14(77.8 \%) \\
3(16.7 \%) \\
1(5.6 \%)\end{array}$ & 7.729 & 0.021 & S \\
\hline Soda cans & $\begin{array}{c}\text { No exposure } \\
\text { Mild expousre } \\
\text { Severe exposure }\end{array}$ & $\begin{array}{c}0(0.0 \%) \\
10(31.3 \%) \\
22(68.8 \%)\end{array}$ & $\begin{array}{c}2(11.1 \%) \\
16(88.9 \%) \\
0(0.0 \%)\end{array}$ & 23.291 & 0 & HS \\
\hline Plastic containers & $\begin{array}{c}\text { No exposure } \\
\text { Mild expousre } \\
\text { Severe exposure }\end{array}$ & $\begin{array}{c}0(0.0 \%) \\
0(0.0 \%) \\
32(100.0 \%)\end{array}$ & $\begin{array}{c}0(0.0 \%) \\
3(16.7 \%) \\
15(83.3 \%)\end{array}$ & 5.674 & 0.017 & S \\
\hline Detr. Clean & $\begin{array}{c}\text { No exposure } \\
\text { Mild expousre } \\
\text { Severe exposure }\end{array}$ & $\begin{array}{c}0(0.0 \%) \\
6(18.8 \%) \\
26(81.3 \%)\end{array}$ & $\begin{array}{c}2(11.1 \%) \\
10(55.6 \%) \\
6(33.3 \%)\end{array}$ & 12.565 & 0.002 & HS \\
\hline Insecticides & $\begin{array}{c}\text { No exposure } \\
\text { Mild expousre } \\
\text { Severe exposure }\end{array}$ & $\begin{array}{c}0(0.0 \%) \\
12(37.5 \%) \\
20(62.5 \%)\end{array}$ & $\begin{array}{c}5(27.8 \%) \\
13(72.2 \%) \\
0(0.0 \%)\end{array}$ & 22.917 & 0 & HS \\
\hline Cosmetics & $\begin{array}{l}\text { No exposure } \\
\text { Mild expousre } \\
\text { Severe exposure }\end{array}$ & $\begin{array}{c}14(43.8 \%) \\
18(56.3 \%) \\
0(0.0 \%)\end{array}$ & $\begin{array}{c}0(0.0 \%) \\
11(61.1 \%) \\
7(38.9 \%)\end{array}$ & 20.366 & 0 & HS \\
\hline Deodrants & $\begin{array}{c}\text { No exposure } \\
\text { Mild expousre } \\
\text { Severe exposure }\end{array}$ & $\begin{array}{c}20(62.5 \%) \\
12(37.5 \%) \\
0(0.0 \%)\end{array}$ & $\begin{array}{c}0(0.0 \%) \\
6(33.3 \%) \\
12(66.7 \%)\end{array}$ & 32.639 & 0 & HS \\
\hline
\end{tabular}




\section{DISCUSSION}

Results of the present study showed highly significant association between age of the patient, BMI of the patient and previous mammographic examinations with occurrence of benign and malignant lesions, axillary lymphadenopathy and breast calcifications. These findings are close to that mentioned by Chalasani (2017) who stated that increasing age is an established risk factor for breast cancer.

Our results were matching with the results obtained by Goehring and Morabia (1997) and Friedenreich et al., (2000) who stated that obesity has been identified as one of the only consistent risk factors for BBD.

Our results are not matching with the results obtained by other investigators as Hislop et al., (1990), Soini et al., (1981), Brinton et al., (1981), Bianchi et al., (1993) and Cole et al., (1978) who mentioned that Obesity has been consistently shown to decrease risk of BPBD. Our results agreed with the results of Chalasani (2017) who mentioned that increased risk of postmenopausal breast cancer has been consistently associated with adult weight gain of 20-25 kg above body weight at age 18 .

Considering relation between alcohol intake and breast diseases, our study showed a significant association between alcohol intake and breast calcifications. There was no significant correlation between alcohol intake and axillary lymphadenopathy and occurrence of benign lesions and occurrence of malignant lesions. These results contradict with the results of many other investigators as Chalasani (2017) who mentioned that increased risk of postmenopausal breast cancer has been consistently associated with regular, moderate consumption of alcohol (3-5 alcoholic beverages per week). 
Coutelle et al., (2004) and Fan et al., (2000) and Fucic et al., (2012) mentioned that Alcohol is related with increased risk of breast cancer development as even low alcohol consumption increases serum estradiol. In a study made on animal model, alcohol increases estradiol levels in dams, which leads to higher levels of ER alpha receptors in their offspring mammary gland and may launch tumori genesis (Hilakivi et al., 2004).

Our results were similar to those mentioned by Rohan \& Cook (1989) and Friedenreich et al., (2000) who found no association between alcohol consumption and BPBD, even after stratification by degree of atypia.

Results of our study showed significant association between caffeine intake and enlarged axillary lymph nodes. No association found between caffeine intake and breast calcifications and occurrence of benign and malignant lesions. Our findings are close to those obtained by Rohan et al. (1989) and Boyle et al. (1984) who found no strong or consistent association between caffeine consumption and the potential deleterious effect on BPBD. Moreover, Webb et al. (2004) stated that caffeine restriction may improve symptoms of BBD. Morrow (2000) mentioned that caffeine avoidance has been a popular treatment measure in women with breast pain. Unfortunately, two randomized clinical trials by Ernster et al. (1982) and Allen \& Froberg (1987) failed to demonstrate a therapeutic benefit for caffeine restriction.

Friedenreich et al. (2000) mentioned that no clear associations with BPBD were observed for some factors as caffeine intake.

Our results showed a high significant association between smoking and enlarged axillary lymph nodes and breast calcifications. Meanwhile, no 
significant association was found between smoking and occurrence of benign and malignant lesions. This was similar to previous studies by $Y u$ et al. (1992) and Berkowitz et al. (1985) who have shown no substantial effect of ever, former or current smoking; however one study by Pastides et al. (1987) found decreased risks and another study by Nomura et al. (1977) showed increased risks for BPBD associated with smoking. In this study, cigarette smoking as assessed as current, past, or passive smoking was not associated with an increased risk of BPBD. Friedenreich et al. (2000) mentioned that no clear associations with BPBD were observed for some factors as smoking.

Our study showed significant association between use of diet and soda cans, use of detergents and cleaning agents, use of insecticides, use of deodorants and use of cosmetics and occurrence of benign and malignant lesions, axillary lymphadenopathy and breast calcifications. Significant association was found between use of plastic containers and occurrence of benign and malignant lesions.

This was matching with Andersen et al. (2006) and Calafat et al. (2008) who stated that BPA is commonly found in polycarbonate plastic products including baby bottles, water bottles, food containers, in the linings of metal food cans and in dental sealants and composites. BPA has been found in over $90 \%$ of the U.S. population age six and over, with highest concentrations in children ages 6-11.

Our results was similar to results published by Lankester et al. (2013), who mentioned that Triclosan is commonly found in antibacterial hand soaps, toothpastes and household cleaning supplies. Phthalate chemicals "soften" plastics to make them pliable. They are also found in personal care products, 
food, plastic toys and household dust. Parabens are found in common personal care products including cosmetics and antiperspirants.

These results agreed with those obtained by Brody \& Rudel (2003) and Brody et al. (2007) who mentioned that currently there are some 160 xenoestrogens that may be involved in breast cancer development. Women are the largest consumers of cosmetic products which may be a significant source of xenoestrogens. Some, such as metalloestrogens (e.g., aluminium salts), parabens, cyclosiloxanes, triclosan, UV screeners, phthalates, Aloe Vera extracts, and musk are present in numerous cosmetics products.

Also Rosenthal et al. (2004) mentioned that humans are exposed to these chemicals transcutaneously and measurable levels have been detected in human breast tissue.

Exposure to environmental estrogens was described by Andersen et al. (2006) who mentioned that Chemicals like polycyclic aromatic hydrocarbons (PAH), pesticides, polychlorinated biphenyl (PCB), dichlorodiphenyltrichlorethane (DDT), some drugs (e.g. antiepileptic drugs), fungicides, cotinine, phytoestrogens, mycotoxins, bisphenol A (a plastics additive), phthalates, alkylphenols, and metalloestrogens mimic estrogen action, affect estrogen levels, or bind to estrogen receptors.

Fucic et al. (2012) stated that Xenoestrogens are present in a number of substrates such as cigarette smoke, automobile exhaust, chemical industry pollutants, grilled meat, volcano dust, forest fire smoke, milk, water, and cosmetic products. This means that all human population may be exposed to them. 


\section{REFERENCES}

Allen, S.S. and Froberg, D.G. (1987): The effect of decreased caffeine consumption on benign proliferative breast disease: a randomized clinical trial. Surgery. 101:720-30.

Andersen, H.R.; Bonefeld-Jorgensen, E.C.; Nielsen, F.; Jarfeldt, K.; Jayatissa, M.N. and Vinggaard, A.M. (2006): Estrogen effects in vitro and in vivo of the fungicide fenarimol. ToxicolLett. 163(2):142-152.

Berkowitz, G.S.; Canny, P.F.; Livolsi, V.A.; Merino, M.J.; O'Connor, T.Z. and Kelsey, J.L. (1985): Cigarette smoking and benign breast disease. J Epidemiol Community Health 39:308-13.

Bianchi, S.; Palli, D.; Galli, M. and Zampi, G. (1993): Benign breast disease and cancer risk. Crit Rev Oncol/Hematol 15:221-42.

Boyle, C.A.; Berkowitz, G.S. and LiVolsi, V.A. (1984): Caffeine consumption and fibrocystic breast disease: a case-control epidemiologic study. J Natl Cancer Inst 72:1015-19.

Brinton, L.A.; Vessey, M.P.; Flavel, R. and Yeates, D. (1981): Risk factors for benign breast disease. Am J Epidemiol 113:203-14.

Brody, J.G.; Rudel, R.A.; Michels, K.B.; Moysich, K.B.; Bernstein, L.; Attfiled, K.R. and Gray, S. (2007): Environmental pollutants, diet, physical activity, body size and breast cancer. Cancer (Suppl) 109(12):2627-2634.

Brody, J.G. and Rudel, R.A. (2003): Environmental pollutants and breast cancer. EHP. 111(8):1007-19.

Calafat, A.M.; Ye, X.; Wong, L.Y.; Reidy, J.A. and Needham, L.L. (2008): Exposure of the U.S. population to bisphenol A and 4-tertiaryoctylphenol: 2003-2004. Environ Health Perspect. 116(1):39-44. doi: 10.1289/ehp.10753.

Cole, P.; Elwood, J.M. and Kaplan, S.D. (1978): Incidence rates and risk factors of benign breast neoplasms. Am J Epidemiol 108:112-20. 
Coutelle, C.; Hohn, B.; Benesova, M.; Oneta, C.M.; Quattrochi, P.; Roth, H.J.; Schmidt-Gayk, H.; Schneeweiss, A.; Bastert, G. and Seitz, H.K. (2004): Risk factor in alcohol associated breast cancer: Alcohol dehydrogenase polymorphism and estrogens. Int $\mathbf{J}$ Oncology. 25(4):1127-1132.

Ernster, V.L.; Mason, L.; Goodson, W.H. $3^{\text {rd }}$; Sickles, E.A.; Sacks, S.T. and Selvin, S. (1982): Effects of caffeine-free diet on benign breast disease: a randomized trial. Surgery 91:263-7.

Evans, K. (2009): Environmental Estrogen: Understand the Problems and Solutions.https://www.naturalnews.com/027729_estrogen_plastic s.html Monday, December, $14^{\text {th }}$. Cited June 2017.

Fan, S.; Meng, Q.; Gao, B.; Grossman, J.; Yadegari, M.; Goldberg, I.D. and Rosen, E.M. (2000): Alcohol stimulates estrogen receptor signaling in human breast cancer cell lines. Cancer Res. 60:56355639.

Friedenreich, C.M.; Bryant, H.E.; Alexander, F. and Hugh, J. (2000): Risk factors for benign proliferative breast disease, Int. J. Epidemiol 29 (4): 637-644

Fucic, A.; MarijaGamulin; ZeljkoFerencic; JelenaKatic; Martin, Krayer von Krauss; Alena, Bartonova and Domenico, F. Merlo (2012): Environmental exposure to xenoestrogens and oestrogen related cancers: reproductive system, breast, lung, kidney, pancreas, and brain. Environ Health. 11(Suppl 1): S8.

Goehring, C. and Morabia, A. (1997): Epidemiology of benign breast disease, with special attention to histologic types. Epidemiol Rev 19:31027.

Hilakivi, C.L.; Cabanes, A.; de Assis, S.; Wang, M.; Khan, G.; Shoemaker, W.J. and Stevens, R.G. (2004): In utero alcohol exposure increases mammary tumorigenesis in rats. Brit $\mathrm{J}$ Cancer. 90:2225-2231.

Hislop, T.G.; Band, P.R. and Deschamps, M. (1990): Diet and histologic types of benign breast disease defined by subsequent risk of breast cancer. Am J Epidemiol 131:263-70. 
Kane, J. (2013): 5 Ways to Reduce Your Exposure to Environmental Estrogens.https://community.breastcancer.org/blog/for-ourmothers-and-others-environmental-estrogens/ May $8^{\text {th }}$. Cited July 2017.

Lankester, J.; Patel, C.; Cullen, M.R.; Ley, C. and Parsonnet, J. (2013): Urinary triclosan is associated with elevated body mass index in NHANES. PLoS One. 8(11):e80057.

Morrow, M. (2000): The Evaluation of Common Breast Problems. Am Fam Physician. 61(8):2371-2378.

Nomura, A.; Comstock, G.W. and Tonascia, J.A. (1977): Epidemiologic characteristics of benign breast disease. Am J Epidemiol 105:505-12.

Pastides, H.; Najjar, M.A. and Kelsey, J.L. (1987): Estrogen replacement therapy and fibrocystic breast disease. Am J Prev Med 3:282-86.

Popli, M.B.; Rahul, Teotia; Meenakshi, Narang and Hare, Krishna (2014): Breast Positioning during Mammography: Mistakes to be Avoided Breast Cancer (Auckl). 8: 119-124.

Rohan, T.E. and Cook, M.G. (1989): Alcohol consumption and risk of benign proliferative epithelial disorders of the breast in women. Int $\mathbf{J}$ Cancer 43:631-36.

Rosenthal, M.D.; Albrecht, E.D. and Pepe, G.J. (2004): Estrogen modulates developmentaly regulated gene expression in the fetal baboon liver. Endocrine. 23(2-3):219-228.

Update: February $22^{\text {nd }}$, Cited June 2017.

Soini, I.; Aine, R.; Lauslahti, K. and Hakama, M. (1981): Independent risk factors of benign and malignant breast lesions. Am J Epidemiol 114: $507-14$.

Yu, H.; Rohan, T.E.; Cook, M.G.; Howe, G.R. and Miller, A.B. (1992): Risk factors for fibroadenoma: case-control study in Australia. Am J Epidemiol 135:247-58. 
Webb, P.M.; Celia, Byrne; Stuart, J. Schnitt; James, L. Connolly; Timothy, W. Jacobs; Heather, J. Baer; Walter, C. Willett and Graham, A. Colditz (2004): A Prospective Study of Diet and Benign Breast Disease, Cancer Epidemiol Biomarkers Prev. 13(7):1106-13.

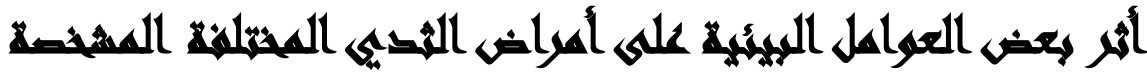

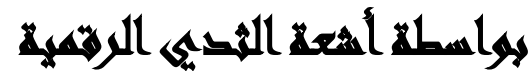

\section{$[r]$}

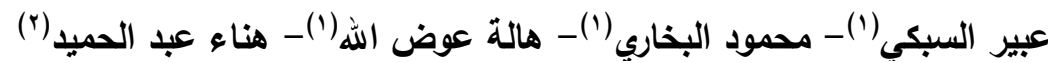

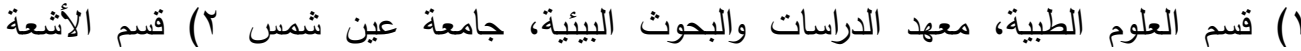

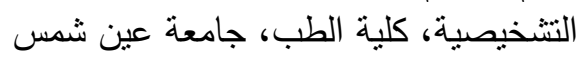

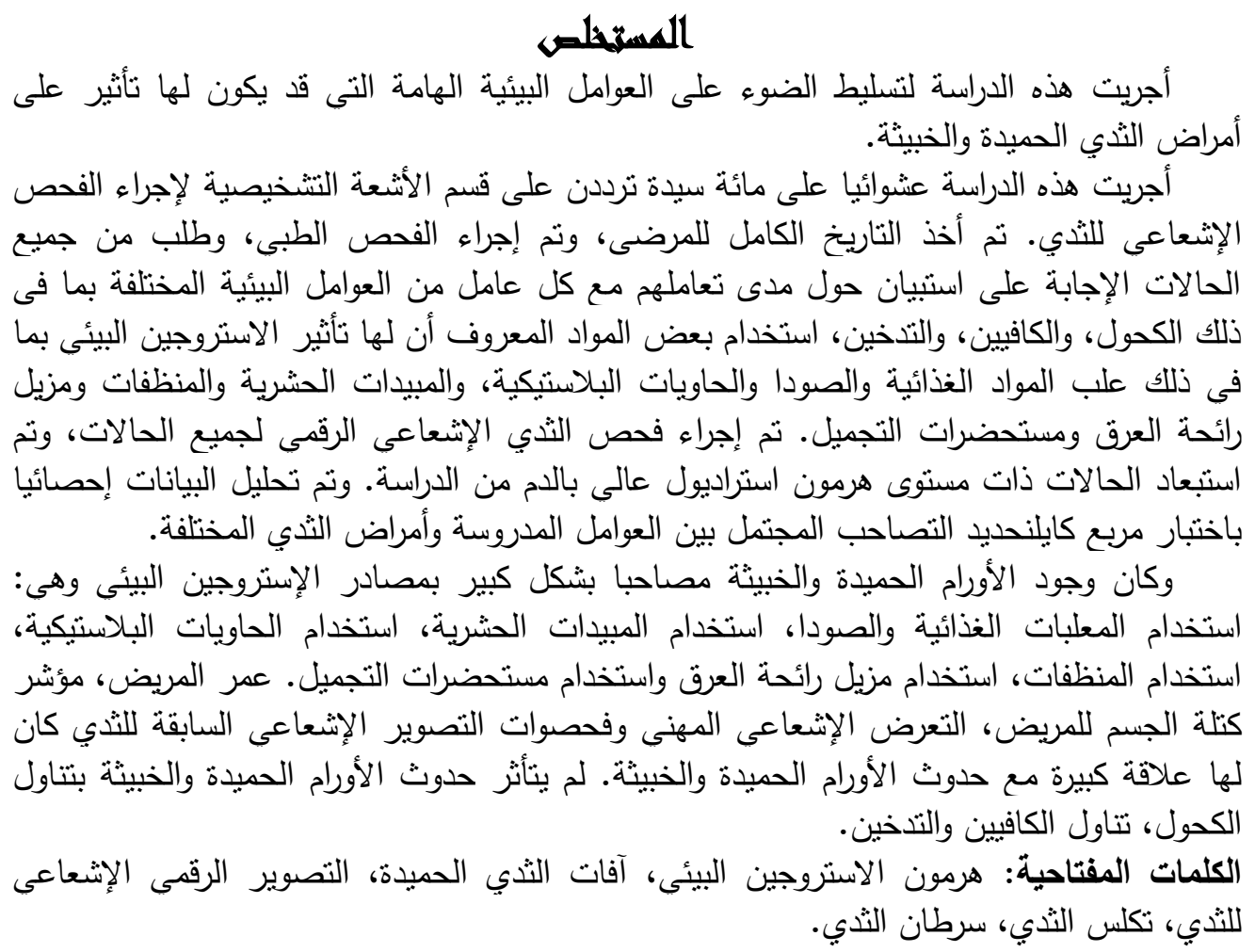

